\title{
Ecthyma gangrenosum caused by Pseudomonas aeruginosa in a patient with astrocytoma treated with chemotherapy
}

\author{
Filip Yves Francine Léon De Vos • \\ Tom Alexander Middelburg • \\ Caroline Seynaeve • Maja J. A. de Jonge
}

Received: 27 May 2009/ Accepted: 23 October 2009/Published online: 7 January 2010

(C) Japanese Society of Chemotherapy and The Japanese Association for Infectious Diseases 2010

\begin{abstract}
Ecthyma gangrenosum, presenting as embolic lesions caused by Pseudomonas aeruginosa infection, has distinct pathognomonic features and a high mortality rate in patients with bacteremia, but when recognized early is easily treated. In this case report we describe this disseminated infection in an adult patient treated with chemotherapy for an astrocytoma.
\end{abstract}

Keywords Ecthyma gangrenosum - Pseudomonas . Hemorrhage · Bullous

\section{Introduction}

Lesions of the skin are common in cancer patients, usually occurring in the context of paraneoplastic skin events such as erythema nodosum and dermatological alterations due to allergic reactions or the side effects of systemic antitumor therapy, or in the context of erysipelas or cellulitis caused by either Streptococcus or Staphylococcus. However, in patients with chemotherapy-induced neutropenia (defined as $<500$ functional polymorphonuclear leukocytes $/ \mu 1$ ), innocent-looking macules or papules may be the first signs of sepsis with uncommon organisms.

F. Y. F. L. De Vos $(\bowtie)$ C. Seynaeve · M. J. A. de Jonge Department of Medical Oncology, Daniel den Hoed Center, Erasmus University, Groene Hilledijk 301,

3075 EA Rotterdam, The Netherlands

e-mail: f.devos@erasmusmc.nl

T. A. Middelburg

Department of Dermatology, Daniel den Hoed Center,

Erasmus University, Rotterdam, The Netherlands
Ecthyma gangrenosum is a rare cutaneous infection with painless, round, necrotic lesions consisting of a central black or gray-black eschar with surrounding erythema. The lesions are usually localized in nonpressure areas (in contrast to ischemic lesions), such as the extremities and gluteal and perineal regions. Solitary lesions have a better prognosis than multiple lesions, the latter being commonly related to life-threatening Pseudomonas aeruginosa bacteremia. Further, the prognosis is determined by an early diagnosis, the duration of neutropenia, and the start of appropriate antibiotic treatment. Predisposing factors, such as chemotherapy, extensive burns, hypogammaglobulinemia, hematological malignancies, and comorbidities (e.g., diabetes mellitus and malnutrition), contribute to the severity of the immunodeficient status, and thus negatively to the outcome [1]. Mortality rates of Pseudomonas sepsis in immunocompromised persons range from 38 to $96 \%$, whereas the mortality rate in nonbacteremic patients is $15.4 \%$ [2]. The reported high mortality rate could be diminished by timely recognition of the early occurring pathognomonic characteristics of ecthyma gangrenosum, so that appropriate systemic antibiotic therapy can be initiated.

\section{Case report}

A 37-year-old Caucasian man with a history of low-grade oligo-astrocytoma in the right temporo-parietal lobe presented in October 2008 with epileptic insults, sensory neuropathy in the left hand, and coordination dysfunction of the left leg. Computed tomography (CT) of the brain showed progressive disease with an increase in size of the known lesion. As radiation therapy had previously been given, with a total dose of up to $50 \mathrm{~Gy}$ on the tumor site, 
this was not an option anymore. Therefore, chemotherapy was opted for, and PVC chemotherapy, consisting of lomustine $110 \mathrm{mg} / \mathrm{m}^{2}$ on day 1 , procarbazine $60 \mathrm{mg} / \mathrm{m}^{2}$ on days $8-21$, and vincristine $1.4 \mathrm{mg} / \mathrm{m}^{2}$ on days 8 and 29 , to be repeated every 6 weeks, was started in November 2008 . Further relevant comedication consisted of dexamethasone $2 \mathrm{mg}$, levetiracetam $1500 \mathrm{mg}$, valproate $1500 \mathrm{mg}$, and clobazam $10 \mathrm{mg}$, all twice daily. On the evening of day 40 of the first course, he was admitted with febrile neutropenia and cold chills. He had no worsening of his neurological symptoms. There were no localizing complaints. At physical examination blood pressure measured $139 / 69 \mathrm{mmHg}$ with a pulse of 109 beats per min and a temperature of $38.5^{\circ} \mathrm{C}$. On his arms, legs, abdomen, and pubic area several hemorrhagic bullous lesions with surrounding erythema were observed, some with central black necrosis (Figs. 1, 2 ). There were no other physical signs of possible infection found. Laboratory results showed $6.6 \mathrm{mmol} / \mathrm{l}$ hemoglobin (normal values $8.6-10.5 \mathrm{mmol} / \mathrm{l}$ ), less than $0.05 \times 10^{9} / 1$ neutrophils (normal values $1.4-8 \times 10^{9} / 1$ ), and $63 \times 10^{9} / 1$ thrombocytes (normal values $150-370 \times$ $10^{9} / \mathrm{l}$ ), with $60 \mathrm{mg} / \mathrm{dl} \mathrm{C}$-reactive protein (maximum value in hospitalization period $170 \mathrm{mg} / \mathrm{dl}$; normal values 0-9 mg/dl). Syndrome of inappropriate antidiuretic hormone secretion by central nervous malignancy was observed, with $125 \mathrm{mmol} / \mathrm{l}$ sodium (normal values $136-145 \mathrm{mmol} / \mathrm{l}$ ) and $260 \mathrm{mOsm} / \mathrm{kg}$ serum osmolality (normal values 270-300 mOsm $/ \mathrm{kg}$ ). After several pictures of the skin lesions had been put on a secure intranet link, a dermatologist was consulted by phone, and suggested sampling bacterial cultures of skin and bullous fluid. Bacteriological sampling was taken from blood, urine, skin, and bullous fluid. Because of the patient's neutropenic fever, we started imipenem/cilastatin 500/500 mg four times daily, intravenously, after the sampling. A day later, the consulted dermatologist examined the patient. At that time, the clinical picture, combined with positive culture of Pseudomonas aeruginosa, was typical for the diagnosis of ecthyma gangrenosum caused by disseminated Pseudomonas infection, and therefore histology of the skin lesions was not obtained. The antibiotic treatment was switched from imipenem/cilastatin to ceftazidime $1 \mathrm{~g}$ six times a day and tobramycin $7 \mathrm{mg} / \mathrm{kg}$ according to tobramycin blood levels, given intravenously [3, 4]. The clinical condition improved after 3 days of hospitalization, the fever subsided after 1 day of this antibiotic treatment, and the neutrophils were restored to a normal level in 9 days. After 1 week, the antibiotics were switched to ciprofloxacin $750 \mathrm{mg}$ bid for 1 week, given orally, and the patient could be discharged. In the second chemotherapy course, lomustine and procarbazine were reduced to $75 \%$ of the original dose, and the patient remained without a recurrent neutropenic episode.

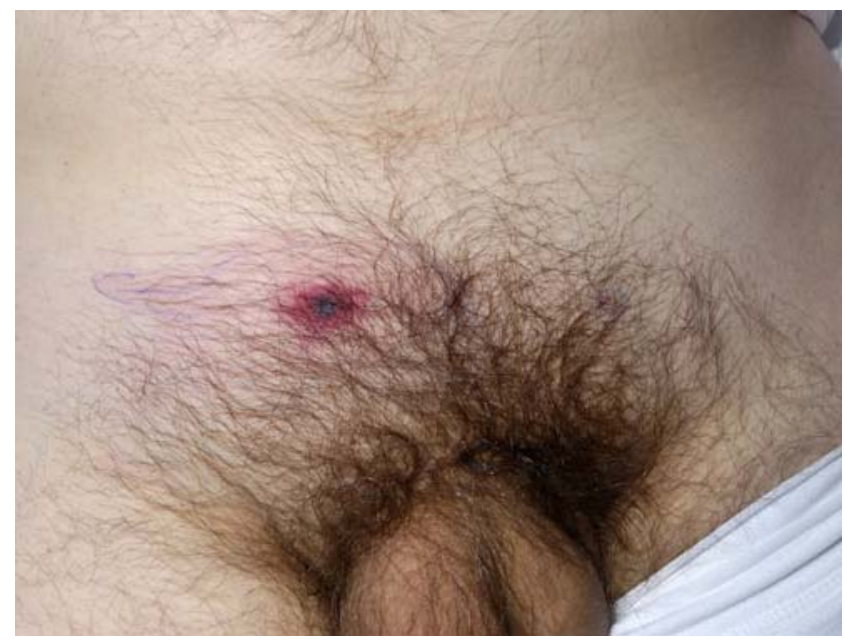

Fig. 1 Lesion with central black necrosis and surrounding erythema in pubic area

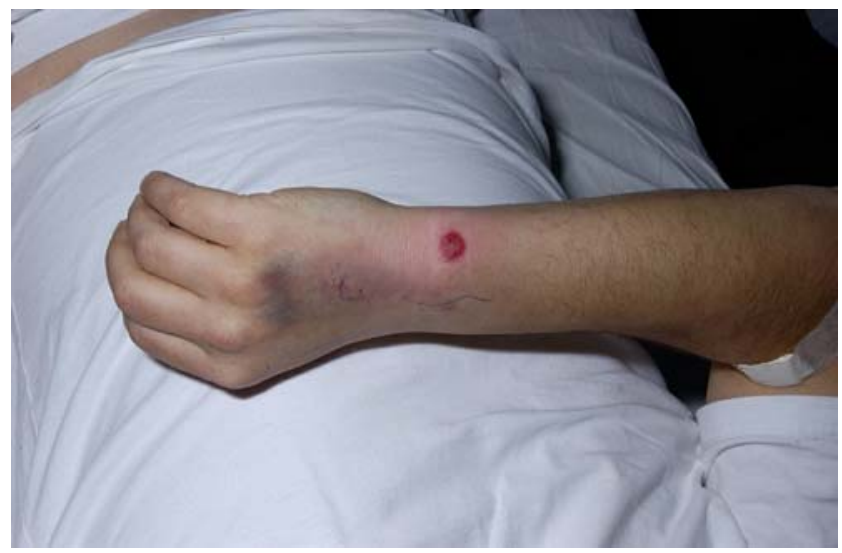

Fig. 2 Hemorrhagic bullous lesion with surrounding erythema on the wrist

\section{Discussion}

Ecthyma gangrenosum is a cutaneous entity that is classically associated with septicemia and gram-negative bacteremia caused by Pseudomonas aeruginosa in immunocompromised patients. Usually, this disseminated cutaneous infection presents with embolic lesions with the formation of bullae. The exact mechanism of pathogenesis of this disease in a neutropenic patient is poorly understood. The Pseudomonas organism releases a variety of proteases in outer-membrane derived vesicles, regulated by a quorum sensing system. The virulence of these pathogenic factors leads to invasion of the medial and adventitial layers of the vascular wall of nearby blood vessels, resulting in septicemia, bacteremia, embolization, and dissemination [5, 6]. After invasion of the subcutaneous tissue, the bacilli are usually found in the collagen bundles of the dermis and panniculus [7]. Proliferation of the 
organisms leads to the production and release of exotoxin $\mathrm{A}$ and proteases, resulting in the formation of bullae and ulceration. As the diagnosis in our patient was already clear from the positive bacterial culture of the bullous fluid, histology was not performed, but would typically show necrotizing hemorrhagic vasculitis with bacterial infiltration into the media and adventitia of vessels resulting in skin necrosis. The lesions are usually situated in the gluteal and perineal region, but can spread to the trunk and extremities and in extreme cases even to the face. Differential diagnosis includes pyoderma gangrenosum, other forms of vasculitis, and the presence of cryoglobulins or septic emboli from other microorganisms (Escherichia coli, Aeromonas hydrophilia, Pseudomonas cepacia, Candida, Fusarium species, and Aspergillus species).

Major risk factors for the development of ecthyma gangrenosum, such as chemotherapy, extensive burns, hypogammaglobulinemia, hematological malignancies, and comorbidities (e.g., diabetes mellitus and malnutrition), are all associated with an immunocompromised condition [2]. The combination of these different risk factors contributes to a life-threatening situation with a high mortality rate in the case of a systemic infection. Mortality rates vary from 38 to $96 \%$ in patients with bacteremia, and are approximately $15 \%$ in patients with a nonbacteremic condition. Multiple skin lesions predict a worse prognosis and can be diagnosed in both septicemic and nonsepticemic patients. However, ecthyma gangrenosum caused by Pseudomonas aeruginosa can develop in only a small number of cases in the absence of bacteremia $[8,9]$. The recommended treatment for both bacteremic and nonbacteremic ecthyma gangrenosum combines an anti-pseudomonas $\beta$-lactam antibiotic or a third-generation cephalosporin with an aminoglycoside, sometimes even with surgical debridement $[3,4]$.

The diagnosis in our patient was made within less than $48 \mathrm{~h}$. This, in combination with an early alert of the patient concerning the febrile episode, may have explained the fact that blood cultures remained negative. Another explanation may be the small number of bacilli present in the bloodstream needed to induce a systemic infection. As ecthyma gangrenosum has a distinctive presentation, a favorable outcome can be achieved when it is treated in a timely fashion with appropriate antibiotics.

As far as we know, ecthyma gangrenosum has mostly been reported in leukemic patients or children with solid tumors. In one study of a small series of women with breast cancer, and in another retrospective study of 111 patients with various solid tumors, ecthyma gangrenosum was described in patients being treated with chemotherapy $[10,11]$. The retrospective study revealed a declining incidence of ecthyma gangrenosum in these patients over the course of years. Our case report reminds the reader of this disseminated infection in an adult patient being treated with chemotherapy for an astrocytoma. A number of risk factors, i.e., a combination of chemotherapy and dexamethasone leading to a neutropenic period of 1 week, culminated in this case of ecthyma gangrenosum. As in our case, timely recognition is important; in our patient this was possible by early culturing and recognition of the clinical picture so that adequate measures could be taken subsequently.

\section{References}

1. Ng W, Tan CL, Yeow V, Yeo M, Teo SH. Ecthyma gangrenosum in a patient with hypogammaglobulinemia. J Infect. 1998; 36:331-5.

2. Huminer D, Siegman-Igra Y, Morduchowicz G, Pitlik SD. Ecthyma gangrenosum without bacteremia. Report of six cases and review of the literature. Arch Intern Med. 1987;147:299-301.

3. Puthucheary SD, Goldsworthy PJ. Ceftazidime and cefotaximea clinician's choice. Clin Ther. 1989;11:186-204.

4. Rolston KV, Bodey GP. Pseudomonas aeruginosa infection in cancer patients. Cancer Invest. 1992;10:43-59.

5. Bomberger JM, Maceachran DP, Coutermarsh BA, Ye S, O'Toole GA, Stanton BA. Long-distance delivery of bacterial virulence factors by Pseudomonas aeruginosa outer membrane vesicles. PLoS Pathog. 2009;5:e1000382.

6. Girard G, Bloemberg GV. Central role of quorum sensing in regulating the production of pathogenicity factors in Pseudomonas aeruginosa. Future Microbiol. 2008;3:91-106.

7. Greene SL, Su WPD, Muller SA. Ecthyma gangrenosum: report of clinical, histopathologic, and bacteriologic aspects of eight cases. J Am Acad Dermatol. 1984;11:781-7.

8. Singh TN, Devi KM, Devi KS. Ecthyma gangrenosum: a rare cutaneous manifestation caused by Pseudomonas aeruginosa without bacteraemia in a leukaemic patient-a case report. Indian J Med Microbiol. 2005;23:262-3.

9. Song WK, Kim YC, Park HJ, Cinn YW. Ecthyma gangrenosum without bacteraemia in a leukaemic patient. Clin Exp Dermatol. 2001;26:395-7.

10. Chatzinikolaou I, Abi-Said D, Bodey GP, Rolston KV, Tarrand JJ, Samonis G. Recent experience with Pseudomonas aeruginosa bacteremia in patients with cancer: retrospective analysis of 245 episodes. Arch Intern Med. 2000;160:501-9.

11. el Baze P, Thyss A, Vinti H, Deville A, Dellamonica P, Ortonne JP. A study of 19 immunocompromised patients with extensive skin lesions caused by Pseudomonas aeruginosa with or without bacteremia. Acta Derm Venereol. 1991;71:411-5. 